\title{
Correction to: Kernel-based collocation methods for Zakai equations
}

\section{Yumiharu Nakano ${ }^{1}$}

Published online: 20 November 2019

(c) Springer Science+Business Media, LLC, part of Springer Nature 2019

\section{Correction to: Stoch PDE: Anal Comp (2019) 7:476-494 https://doi.org/10.1007/s40072-019-00132-y}

The claim of Lemma 3.7 in [1] needs to be modified. This lemma states that the sum of the absolute value of the cardinal functions is bounded with respect to the approximation parameters and the spatial variables. This result is inconsistent with the mean value theorem in the one dimensional case. The proof of the lemma heavily relies on Assumption 3.4 (ii) in [1], which is only numerically validated in the paper.

In the corrected version, Assumption 3.4 (ii) in [1] is replaced by a weaker condition, and the claim of Lemma 3.7 is replaced by the inequality for the only finite number of points, rather than for all points in the approximation region. Then, there causes no inconsistency in this version. According to these changes, several points in [1] are revised as follows:

(i) The notation $\Delta_{2} x$ is no longer used and so deleted.

(ii) In Theorem 3.4, the error estimation is done for the collocation and evaluation points, rather than for all points in the approximation region.

(iii) Lemma 3.5 is no longer used and so deleted.

(iv) The numerical experiment in Section 4 is replaced with a new one for validating the revised assumption.

Also, the proof of Lemma 3.7 in [1] is simplified at this occasion, where Whitney's extension theorem is used.

The original article can be found online at https://doi.org/10.1007/s40072-019-00132-y.

Yumiharu Nakano

nakano@c.titech.ac.jp

1 Department of Mathematical and Computing Science, School of Computing, Tokyo Institute of Technology, W8-28, 2-12-1, Ookayama, Meguro-ku, Tokyo 152-8550, Japan 


\section{Reference}

1. Nakano, Y.: Kernel-based collocation methods for Zakai equations. Stoch. Partial Differ. Equ. Anal. Comput. 7, 476-494 (2019)

Publisher's Note Springer Nature remains neutral with regard to jurisdictional claims in published maps and institutional affiliations. 